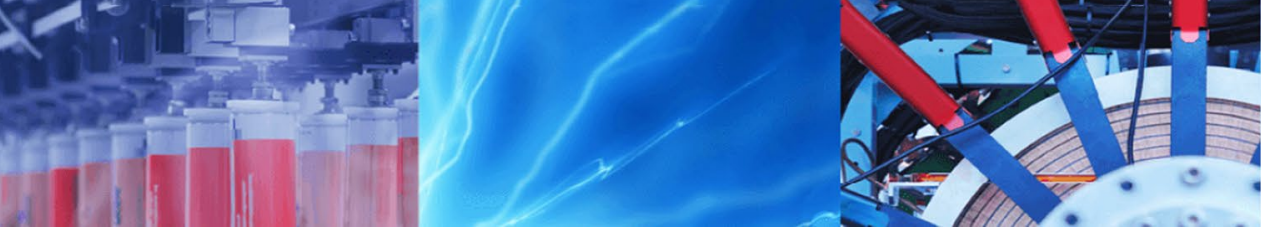

Research Article

\title{
Wearable supercapacitors based on graphene nanoplatelets/carbon nanotubes/polypyrrole composites on cotton yarns electrodes
}

\author{
Ravi Moreno Araújo Pinheiro Lima ${ }^{1} \cdot$ Mário César Albuquerque de Oliveira ${ }^{1}$ Helinando Pequeno de Oliveira ${ }^{1}$ (D)
}

(c) Springer Nature Switzerland AG 2019

\begin{abstract}
The development of highly conductive, flexible, mechanical reinforced and chemically modified cotton yarns for electrodes of supercapacitors represents an important advance in the energy storage devices applied in wearable electronics. The production of carbon-based conductive layers as supports for chemical polymerization of active polymeric materials (such as polypyrrole) is an important strategy that associates the high electrical double-layer capacitance of the carbon derivatives (carbon nanotubes and graphene nanoplatelets) and the pseudocapacitance of the polypyrrole in truly flexible devices with improved electrochemical response-high capacitance. These properties are affected by relative concentration of graphene nanoplatelets in carbon complexes due to the variation in overall conductivity of electrodes (in consequence of low aggregation degree and available surface area) and the electrochemical properties of the resulting devices that reaches capacitance in order of $45.5 \mathrm{~F} \mathrm{~g}^{-1}$ with a capacitive retention of $70 \%$ after 2000 cycles of use. These promising results open possibilities for new systems in wearable electronics.
\end{abstract}

Keywords Supercapacitors · Wearable electronics · Graphene · Carbon nanotubes · Polypyrrole

\section{Introduction}

The widespread application of wearable electronics in healthcare devices, actuators and sensors requires truly wearable devices [1-8]. In consequence, it has been observed an increasing demand for lightweight and flexible devices characterized by high conductivity level, thermal stability and negligible degradation under repeated use [9]. One of the most important applications for wearable devices refers to the development of flexible storage devices (flexible supercapacitors) [10] with characteristic high-power density, long cycling life and fast charge-discharge rate $[1,2,11-13]$.

The advances in flexible and stretchable energy storage devices must be focused on production of more efficient electrodes with improved electrochemical performance of power/energy density, in response of new concepts about electrodes, current collectors and packaging materials
[14]. Flexible supercapacitors are classified in three different types: porous three-dimensional structures, paperlike and fiber-like devices [15]. Paper-like supercapacitors make use of cellulose-based surfaces filled with electrochemically active and conductive materials [16]. In common with the 3D porous structures, the most important point to be addressed in these structures refers to the reduction in electrochemically "dead weight" of supports that presents almost negligible gravimetric capacitance [17]. In view of this, the fiber-like supercapacitors explore lightweight flexible supports disposed in two different configurations: the type I in which two fibers are twisted around each other and the type 2 that makes use of coaxial structure-inner and outer electrodes separated by an electrolyte [15].

It is worth mentioning that low performance in energy density $(E)$ remains as a drawback to be circumvented in the development of wearable supercapacitors.

Helinando Pequeno de Oliveira, helinando.oliveira@univasf.edu.br | ${ }^{1}$ Institute of Materials Science, Federal University of Sao Francisco Valley, Juazeiro, BA 48920-310, Brazil.

SN Applied Sciences (2019) 1:325 | https://doi.org/10.1007/s42452-019-0343-5

Received: 11 January 2019 / Accepted: 5 March 2019 / Published online: 9 March 2019 
If considered that energy is given by $E=\frac{1}{2} C V^{2}$, there are two possibilities to be addressed: the extension in working potential window or the increase in the specific capacitance [12]. The improvement in the electrochemical properties (specific capacitance) depends on chemical modification of electrodes.

Different flexible supports have been explored in the chemical modification of electrodes for incorporation of electrochemical active materials (such as activated carbon fiber felt [18], air-laid paper substrate [19] and elastics cords [20]). To confer small resistance for electron and ion movement along electrodes, it has been reported different strategies for chemical modification of surfaces that varies from simple impregnation of carbon nanotubes and graphene on activated carbon fiber felt [18], dipping and drying method for CNT incorporation followed by in situ chemical polymerization of polyaniline [19] and electrochemical deposition of polyaniline on overlaid CNT layer on elastic cord [20].

Textile-based electrodes offer flexibility and porosity degree required for wearable devices. However, their characteristic poor electrochemical performance remains as a serious drawback to be circumvented [18]. For this, the incorporation of carbon nanofillers (carbon nanotubes and graphene) endows high electrical conductivity and good electrochemical performance for electrodes [21] allowing the additional advantage for use as breathable supercapacitors [22]. In both cases, the association of charge accumulation processes at CNT and graphene surfaces with fast redox reactions on conducting polymers and metal oxides tends to improve the performance of the electrochemical flexible devices [23]. On the other hand, an important point to be addressed refers to the serious aggregation process that takes place at high concentration of carbon nanotubes in flexible supports [18].

In terms of natural flexible supports, the cotton is widely applied in textiles, characterized by high available surface area and high mass loading capability. Despite these important properties, the cotton yarn is characterized by poor electrical properties (it is an insulator) [24, 25]. Cotton-based supercapacitors require adequate covering of cotton yarns with electrochemically active materials, that offer both higher energy and power density in response of adequate combination of electric double-layer capacitors (EDLC) and pseudocapacitors $[1,2,26]$.

Graphene (a very promising EDLC system) is a honeycomb lattice of $s p^{2}$-hybridized carbon atoms that offers high surface area, high thermal and electrical conductivity that can be combined with different materials $[1,27]$, providing core-shell structures (with pseudocapacitors, as an example) for different devices [1, 12]. As an alternative, carbon nanotubes represent other important class of materials for use as EDLC supports, with advantages relative to their simple functionalization [13]. In terms of candidates for use as pseudocapacitors, polypyrrole has been considered an important material for flexible supercapacitors due to its high conductivity and chemical stability provided by chemically synthesized polymeric chains on flexible substrates $[11,13,26,28-30]$. The adequate covering of cotton yarns by carbon derivatives (carbon nanotubes-CNT and graphene) followed by chemical polymerization of conducting polymers renders flexible materials with improved properties for energy storage applications [24, 31-34].

With the aim of optimizing both surface area for charge accumulation and high conductivity level for electrodes, we explored the variation in the relative concentration of carbon derivatives (multiwall carbon nanotubes and graphene nanoplatelets) applied as a cover layer on cotton yarn for deposition of active layer of polypyrrole. Based on the variation of electrical conductivity of resulting flexible electrodes, we established the most adequate combination of carbon nanotubes and graphene nanoplatelets (GNP) to provide high surface area/conductivity for electrodes and consequently the improvement in specific capacitance of material/energy density of device. To evaluate the more adequate ratio of GNP/CNT (EDLC contribution) on cotton yarn, we prepared samples with different ratio of GNP/CNT (10/90, 25/75, 50/50, 75/25 and 90/10) from layer deposition process (in a number of five layers) for incorporation of additives on cotton yarns. The following step (interfacial polymerization of polypyrrole) results in the deposition of a polymeric layer of pseudocapacitor on EDLC support that improves their electrochemical performance and retention of electrochemical properties under mechanical efforts.

\section{Experimental}

\subsection{Materials}

Graphene nanoplatelets (GNP), multiwall carbon nanotubes (CNT), pyrrole, ethanol, anhydrous ferric chloride, triton X-100, camphorsulfonic acid (CSA) and dodecyl benzene sodium sulfonate (SDBS) (all from Sigma-Aldrich), hydrochloric acid (Quimica Moderna, Brazil) and hexane (Synth, Brazil) were used as received. Pyrrole was distilled under reduced pressure before each experiment.

\subsection{Pre-treatment of cotton yarns}

An initial step (based on Refs. [35-37]) was considered for impurities removal and established as follows: cotton yarns were immersed in aqueous solution $(10 \mathrm{~mL})$ of triton 
$\mathrm{X}-100(1 \mathrm{~mL})$ and rinsed with Milli-Q water-the samples were dried in an oven $\left(100^{\circ} \mathrm{C}\right)$. Following this procedure, the cotton yarns were kept under sonication in alcohol, acetone and water for 15,5 and $5 \mathrm{~min}$, respectively-with the repetition of the last two steps.

\subsection{Chemical functionalization of carbon nanotubes}

The preparation of carbon ink for impregnation of carbon nanotubes in pretreated cotton yarns was established with an initial step of chemical functionalization of CNTs.

CNT $(2 \mathrm{~g})$ was introduced in acidic solution $(250 \mathrm{~mL})$ of $\mathrm{H}_{2} \mathrm{SO}_{4} / \mathrm{HNO}_{3}$ (3:1) - the resulting solution was kept under intense stirring for $5 \mathrm{~h}$ at $130^{\circ} \mathrm{C}$ under reflux to avoid the solvent evaporation [38]. Then, the solution was cooled at room temperature. After this step, the resulting material was filtered, rinsed few times using Milli-Q water to neutralize the $\mathrm{pH}$ and dried in an oven at $60^{\circ} \mathrm{C}$ for $24 \mathrm{~h}$.

\subsection{Preparation of (GNP/CNT)-modified cotton yarns}

The coating of the cotton yarns with GNP/CNT layers was performed as follows:

The carbon ink was prepared considering a fixed amount of carbon derivatives (total mass of $50 \mathrm{mg}$ ) in $50 \mathrm{~mL}$ of Milli-Q water. The distribution between CNT and
GNP was varied in five different ratios: $90 \%$ GNP-10\% CNT, 75\% GNP-25\% CNT, 50\% GNP-50\% CNT, 25\% GNP-75\% CNT and 10\% GNP-90\% CNT. The dispersion of GNP/CNT mixture was favored by inclusion of SDBS $(0.5 \mathrm{~g})$ and the sonication of resulting solution for $2 \mathrm{~h}$ [38]. The pretreated cotton yarns were immersed into the GNP/CNT ink and kept under sonication for $15 \mathrm{~min}$. The solvent removal was established in an oven $\left(100^{\circ} \mathrm{C}\right)$. After that, the fiber was washed in water to remove the exceeding material and kept in an oven $\left(100^{\circ} \mathrm{C}\right)$. This process (schematically drawn in Fig. 1) was repeated five times resulting in a good covering degree of GNP/CNT on textiles.

\subsection{Synthesis of PPy on (GNP/CNT) cotton yarns}

A first solution containing $3 \mathrm{~mL}$ of water, $0.251 \mathrm{~g}$ of CSA and $0.175 \mathrm{~g}$ of $\mathrm{FeCl}_{3}$ was prepared (aqueous phase-kept under agitation for $5 \mathrm{~min}$ ) and received the cotton yarn for impregnation with oxidant. After that, it was cooled to $3^{\circ} \mathrm{C}$ for $20 \mathrm{~min}$.

A second solution (oil phase) was also prepared using $3 \mathrm{~mL}$ of hexane and $50 \mu \mathrm{L}$ of pyrrole and kept at $3{ }^{\circ} \mathrm{C}$ for $20 \mathrm{~min}$. After this step, the oil phase was slowly verted on aqueous phase and kept at $3^{\circ} \mathrm{C}$ for $12 \mathrm{~h}$. During this procedure, the polymerization takes place and a film of PPy covers the previous carbon layer [13, 30]. Resulting composites of PPy/(GNP/CNT) on cotton yarns were dried

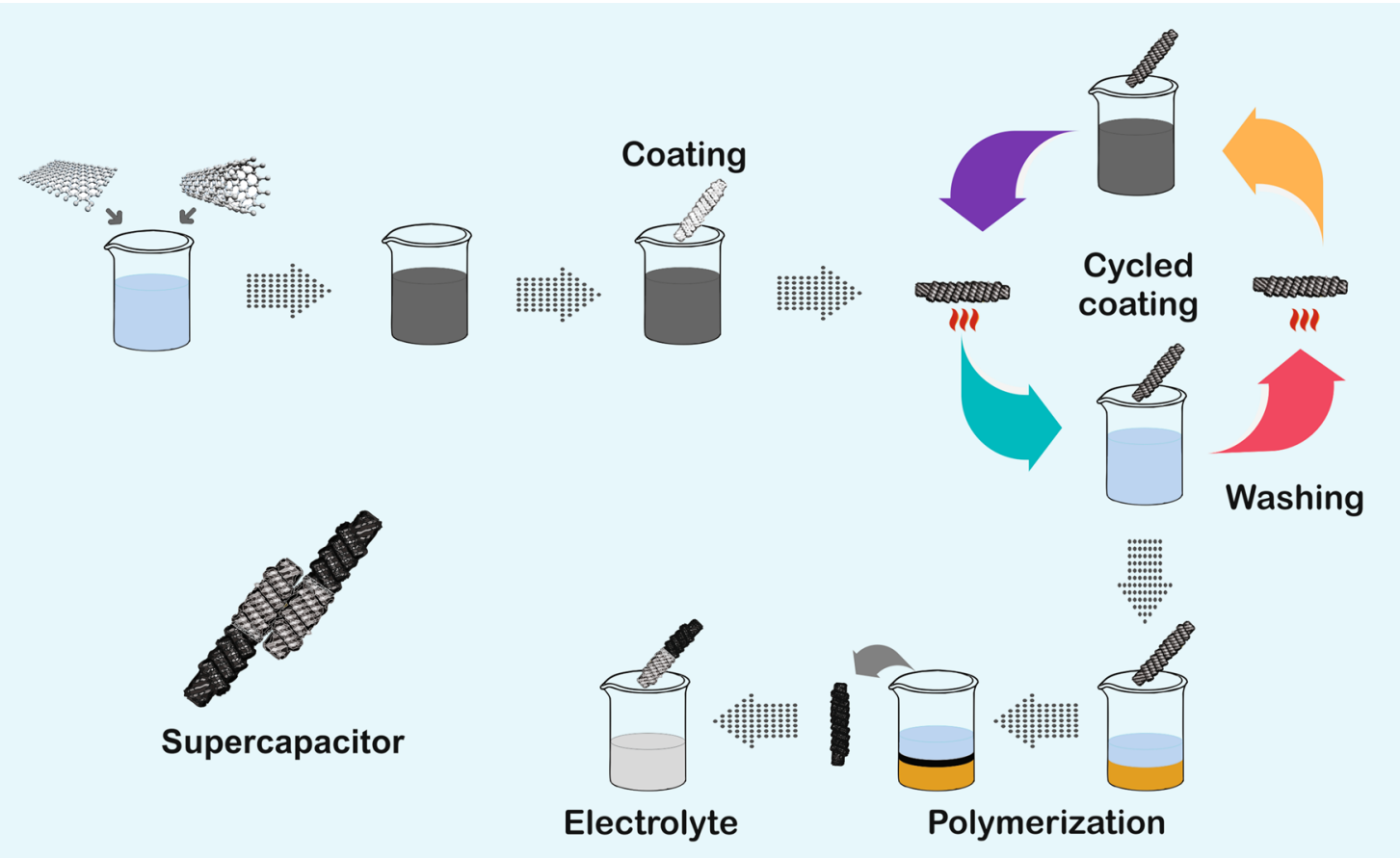

Fig. 1 Schematic view of polymerization process and preparation of polypyrrole-based electrodes 
at $25^{\circ} \mathrm{C}$ and applied as electrodes in the final step for production of supercapacitor prototypes.

\subsection{Preparation of supercapacitor prototypes}

The solid electrolyte to be explored in devices is prepared as follows: polyvinyl alcohol (PVA-1 g) is dispersed in $10 \mathrm{~mL}$ of deionized water. The reactor with PVA solution is introduced in a thermal bath at $90{ }^{\circ} \mathrm{C}$ for $2 \mathrm{~h}$ to completely solubilize the PVA. The solution was left cooling at room temperature and stirred for $15 \mathrm{~min}$, while it was adding dropwise of phosphoric acid ( $1 \mathrm{~mL} \mathrm{H}_{3} \mathrm{PO}_{4}$ at $\left.85 \mathrm{wt} \%\right)$ [39-41].

After this step, pairs of modified cotton yarns $(1 \mathrm{~cm})$ are immersed in the PVA solution for electrolyte impregnation. The resulting electrodes are disposed in parallel with a fixed distance of $1 \mathrm{~mm}$ from each other, as represented in Fig. 1. The device is introduced in an oven at $40^{\circ} \mathrm{C}$ for $10 \mathrm{~min}$ and receives additional droplets of $\mathrm{PVA} / \mathrm{H}_{3} \mathrm{PO}_{4}$ to improve the adhesion degree between electrodes of supercapacitor. Finally, the device is dried in the oven at $40{ }^{\circ} \mathrm{C}$ for $10 \mathrm{~min}$.

\subsection{Characterization techniques}

Scanning electron microscopy (Vega 3XM Tescan at accelerating voltage of $5 \mathrm{kV}$ ) was used to acquire SEM images, while the electrical response (cyclic voltammetry, charge-discharge curves and impedance spectroscopy) of the composites was measured from an Autolab PGSTAT $302 \mathrm{~N}$ (Metrohm)-considering samples of $2 \mathrm{~cm}$. The external DC voltage was provided by a DC Power Supply HY3003-3 (Polyterm). Raman spectra were acquired in a Raman spectrometer (LabRAM Aramis-Horiba Jobin-Yvon) in the range of $800-1800 \mathrm{~cm}^{-1}$ with excitation at $532 \mathrm{~nm}$ from a He-Ne laser with power of $2.5 \mathrm{~mW}$.

\section{Results and discussion}

The morphology of materials prepared by successive deposition of carbon layers and polypyrrole is depicted in Fig. 2, which is compared with the structure of pristine cotton yarns (Fig. 2a), GNP/CNT composite (75\% of GNP) Fig. 2b, c and PPy/(GNP/CNT) (75\% of GNP) (Fig. 2d). As
Fig. 2 SEM images of cottonbased composites: a pristine support, b, c GNP/CNT samples (different magnification) and $\mathbf{d}$ after chemical polymerization of polypyrrole on GNP/CNT layer (PPy/(GNP/CNT))-75\% of GNP
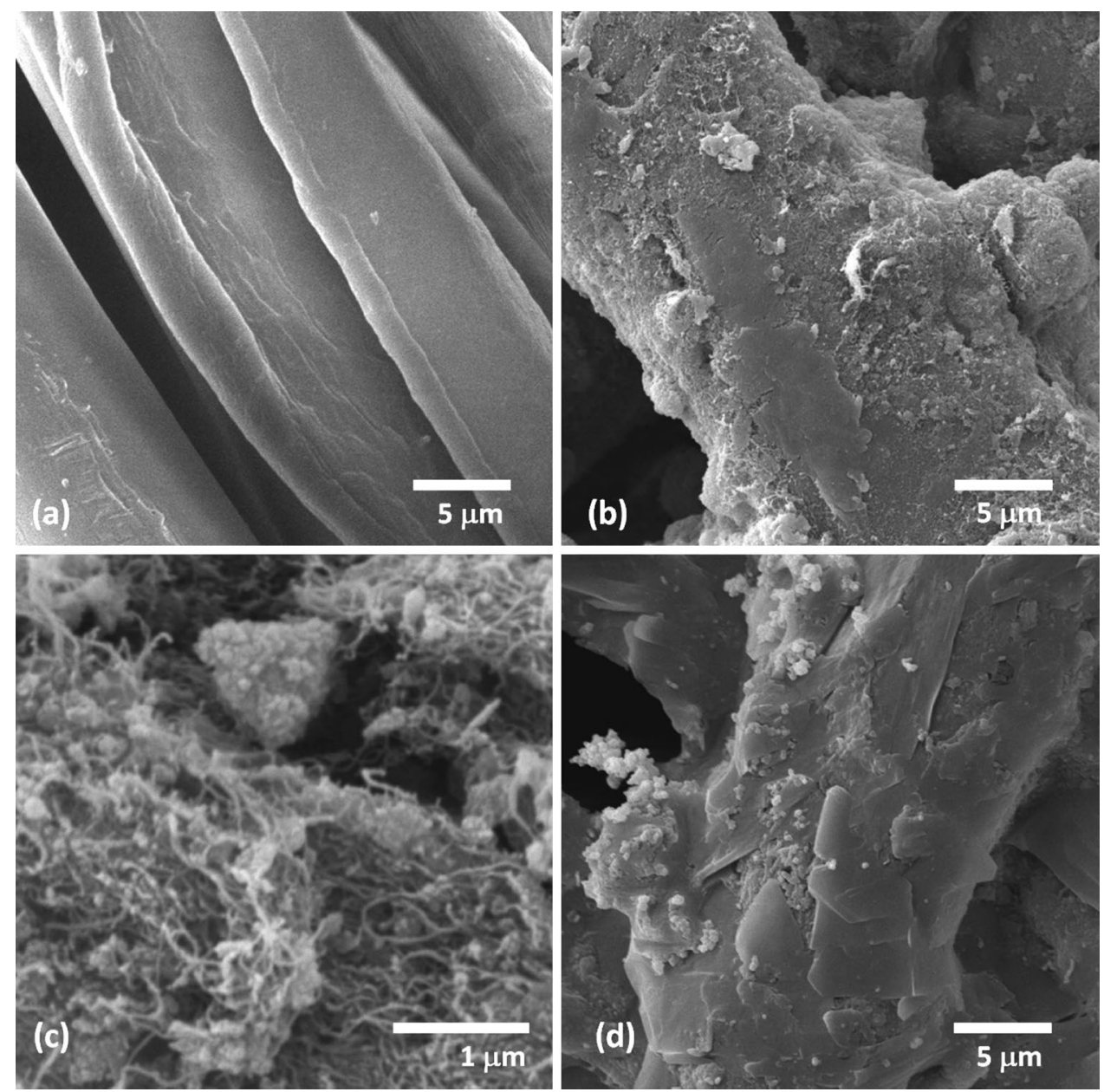
we can see, smooth surface of pristine yarn is modified by incorporation of GNP/CNT that affects the rugosity degree of material. SEM image with higher magnification (see Fig. 2c) reveals the abundance of carbon nanotubes on fiber surface and the dispersion of small grains of graphene as islands in structure of CNTs. The following step (chemical polymerization) results in the complete covering of structure with a thin layer of conducting polymerpolypyrrole-progressively deposited as platelets on previous carbon-based layer. The progressive polymerization favors the growth of polymeric grains on top of polypyrrole layer-as indicated in Fig. $2 d$.

Relevant information concerning to the chemical composition and interaction level of carbon nanotubes and graphene nanoplatelets with polypyrrole can be addressed by Raman spectrum - results are summarized in Fig. 3. The pristine layer of CNT on cotton yarn presents two specific bands: at $1340 \mathrm{~cm}^{-1}$ - the D-band (fingerprint of $s p^{3}$ carbon) characteristic of defects and edges of nanotubes or impurities and $1585 \mathrm{~cm}^{-1}$-and the G-band, related to crystalline graphitic structures-characteristics of in-plane Raman vibration modes of $\mathrm{C}-\mathrm{C}$ bond- $-s p^{2}$ bonded carbon atoms [42].

In correspondence, characteristic bands at $1340 \mathrm{~cm}^{-1}$ (D-band) and $1574 \mathrm{~cm}^{-1}$ (G-band) [43] are observed for GNP. It is worth mentioning that ratio between intensities of D-band and G-band can be explored as an estimative about $s p^{2} / s p^{3}$ atomic ratio, associated to defect concentration degree in carbon structures $[13,26,44$, 45]. Corresponding values calculated for GNP and CNT returned $\left(I_{D} / I_{G}\right)_{G N P}=0.63$ while $\left(I_{D} / I_{G}\right)_{C N T}=1.11$, revealing a higher order for graphene layer than corresponding defective and disordered structure of CNT—the amount of defects can be attributed to the functionalization of carbon nanotubes.

The following step of polymerization on carbon layer introduces three characteristic bands on previously observed D- and G- band: the incorporation of polypyrrole by interfacial polymerization introduces characteristic fingerprints of polypyrrole-bands at $926 \mathrm{~cm}^{-1}$ assigned to $\mathrm{C}-\mathrm{H}$ ring deformation vibration-bipolaron ring deformation [46, 47], at 962 and $1044 \mathrm{~cm}^{-1}$ assigned to polaron symmetric $\mathrm{C}-\mathrm{H}$ in-plane bending vibration [47]. The small displacement observed to lower wavenumber in $\mathrm{D}$ - and $\mathrm{G}$-characteristic bands of carbon derivatives with polypyrrole layer (samples PPy/(GNP/CNT)) characterizes the presence of $\pi-\pi$ interaction between the benzene rings from the CNTs and the aromatic rings of pyrrole, as previously reported [13,26,44]. The comparison of $I_{D} / I_{G}$ values for different concentration of GNP in GNP/CNT complexes is summarized in Fig. $3 \mathrm{~b}$.

In agreement with previously observed behavior for pristine CNT-based samples-high amount of CNT in matrix returns $I_{D} / I_{G}>1$ characterizing the prevailing disorder induced by CNT on overall behavior of samples. However, an important point to be addressed refers to the variation in corresponding value observed for increasing amount of GNP and corresponding number of defects in the resulting matrix. The general trend of reduction in $I_{D} / I_{G}$ continues until to reach a well-defined minimum at relative concentration of GNP/CNT in order of $75 \%$. Above this relative concentration, the disorder prevails and the value of $I_{D} / I_{G}$ assumes an increasing value-characterizing this relative concentration as a critical point in which the disorder in GNP/CNT composite tends to a minimum value.
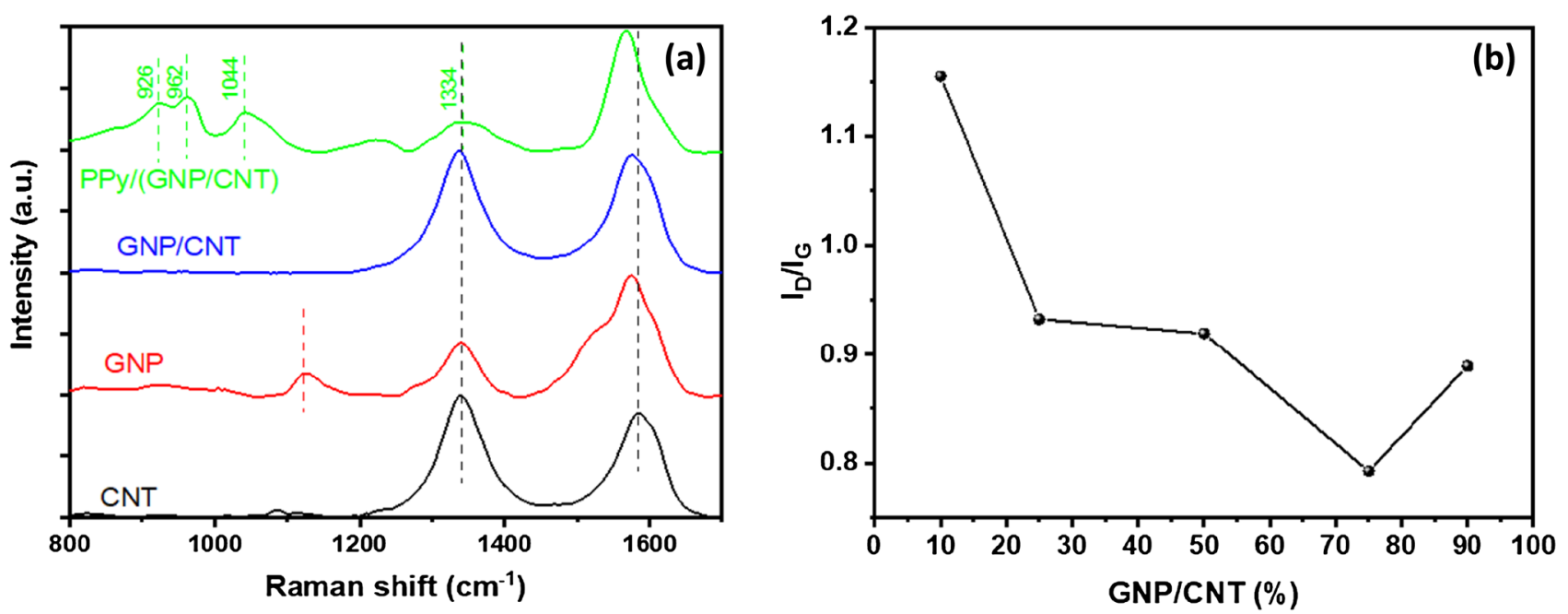

Fig. 3 a Raman spectra of cotton covering layers (CNT, GNP/CNT and PPy/(GNP/CNT)) and b comparison of $I_{D} / I_{G}$ for different GNP/CNT composition 
The electrical response of cotton yarns covered by carbon layer (different relative concentration of GNP/ CNT) shown in Fig. 4a returns a linear dependence (ohmic behavior) for all of samples with increasing slope in the $I-V$ curve associated to increasing concentration of GNP in GNP/CNT mixture (in the range of $10 \%<\mathrm{GNP} / \mathrm{CNT}<75 \%$ ). Above the relative concentration of $75 \%$ of GNP in composition, it is observed a strong decrease in the slope of characteristic $I-V$ curve as consequence of decreasing conductivity of sample.

The polymerization of polypyrrole on carbon layer results in a strong increase of conductivity level of resulting matrix (the current is enhanced by a factor of 30). An important aspect to be observed refers to the dependence of conductivity level of resulting samples with the electrical response of previously deposited layer. The slope of $I-V$ curves increases with relative amount of GNP and reaches a maximum (current in order of $70 \mathrm{~mA}$ ) for sample $75 \%$ GNP. As previously observed, the higher amount of GNP in mixture affects the electrical response of samples. Above this concentration, typical aggregation of GNP tends to reduce the conductivity level of samples-in correspondence with the behavior observed from Raman spectra $\left(I_{D} / I_{G}\right.$ ratio).

Based on these results, corresponding values for conductivity of samples were calculated-data are shown in Fig. 3c. As we can see, the general behavior for conductivity level of PPy-based samples follows the general trend of GNP/ CNT-covered samples. As a consequence, the best conductivity was observed for sample containing $75 \%$ of GNP that reaches $7 \mathrm{~S} \mathrm{~cm}^{-1}$. Consequently, the sample prepared with $75 \%$ of GNP was chosen as the most promising template for supercapacitor electrodes-due to the association of best
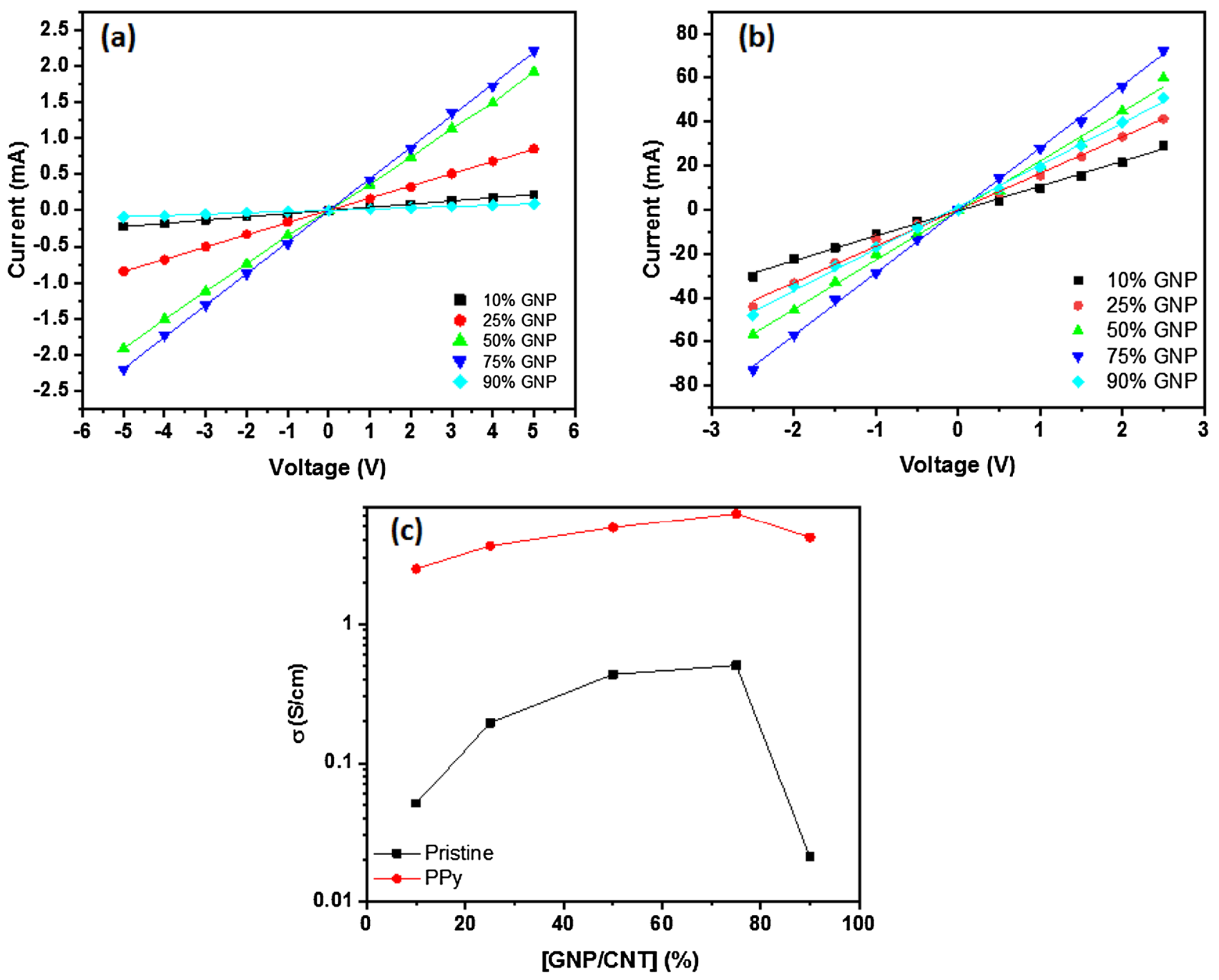

Fig. 4 a $I-V$ curves for GNP/CNT samples at different concentration of GNP/CNT, b $I-V$ curves for PPy/(GNP/CNT) composites at different concentration of GNP/CNT, c dependence of conductivity of samples with composition 
conductivity level of resulting material and lower aggregation level associated to GNP/CNT complexes.

The prototypes of supercapacitors were assembled according Sect. 2.6 using electrodes prepared with $75 \%$ of GNP. Resulting devices were characterized by standard electrochemical methods (galvanostatic charge discharge assays, cyclic voltammetry, impedance and cyclability assays) to evaluate the potential for use as charge storage device.

The cyclic voltammograms shown in Fig. 5a present symmetric curves with cone-shaped behavior at increasing scan rate in substitution to rectangular format of electrical double-layer capacitance. The prolate curves are due to the Faradaic pseudocapacitance of polypyrrole, while the distortion observed at increasing scan rate is a consequence of difference between bulk and surface resistance. Higher scan rate imposes a limitation for available time to promote the diffusion of charge carriers that accumulate at interfaces, prevailing the fast Faradaic mechanisms at external (polypyrrole) layer. The specific capacitance of devices was evaluated by galvanostatic charge-discharge curves of supercapacitor at different current densities. Figure $5 \mathrm{~b}$ summarizes the typical behavior of sample at different current density: the deviation from a straight line in charge and discharge is a consequence of Faradaic prevailing mechanisms. As expected, the IR drop is a current-dependent function while more time of discharge is observed to lower current density. The value of specific capacitance was calculated according Eq. 1 which is based on discharge curves:

$C_{\mathrm{s}}=\frac{2 D_{\text {area }} \cdot I}{V^{2} \cdot m}$

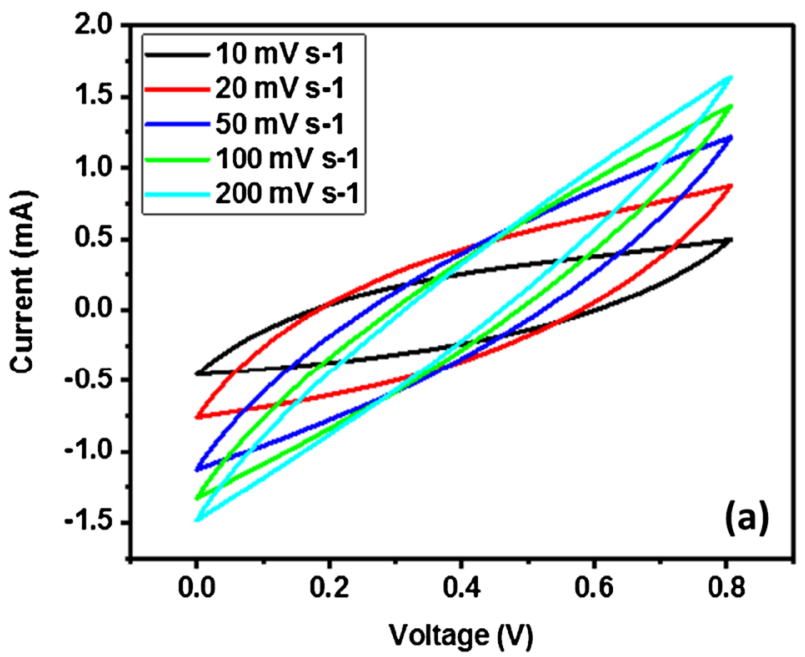

where $V$ is the potential ( $V$ from the IR drop to zero, $I$ is the current of discharge $(A), \mathrm{m}$ is the mass of active layer and $D_{\text {area }}$ is the area under the discharge curve.

The results for specific capacitance are shown in Fig. 6 a and confirm that modulus of capacitance varies inversely with the current density. The maximum in capacitance reaches a maximum of $43.47 \mathrm{~F} \mathrm{~g}^{-1}$ for current of $300 \mathrm{~mA}$. By comparison with previous reported data with pristine CNT structure as support layer [13], it is possible to identify an increase in specific capacitance from 30 to $43.47 \mathrm{~F} \mathrm{~g}^{-1}$ in consequence of two important factors: the higher surface area of graphene derivative for charge accumulation and the reasonable level of conductivity of carbon support layer disposed as a template for polypyrrole growth.

The general behavior observed in Fig. 6a is in agreement with data reported in the literature-the specific capacitance varies inversely with the charging current [13]. From this plot, it is possible to calculate the corresponding maximum in energy density and current density. The corresponding value for energy density is $5.9 \mathrm{~mW} \mathrm{~h} \mathrm{~g}^{-1}$ (in comparison with $\sim 3 \mathrm{~mW} \mathrm{~h} \mathrm{~g}^{-1}$ in Ref. [13].) and for power density in order of $68.75 \mathrm{~mW} \mathrm{~g}^{-1}$ (that is higher than observed for pristine carbon nanotube system $-30 \mathrm{~mW} \mathrm{~g}^{-1}$ - Ref. [13]). These results confirm that incorporation of graphene as a support layer in flexible devices represents a critical factor for improvement in the electrochemical properties of flexible supercapacitors.

Another critical experiment refers to the cyclability of resulting device, applied in the evaluation of devices performance under repeated charge-discharge cycles. For this, the response of supercapacitor was registered along 2000 cycles of continuous charge/discharge. The capacitive retention was calculated as the variation in specific

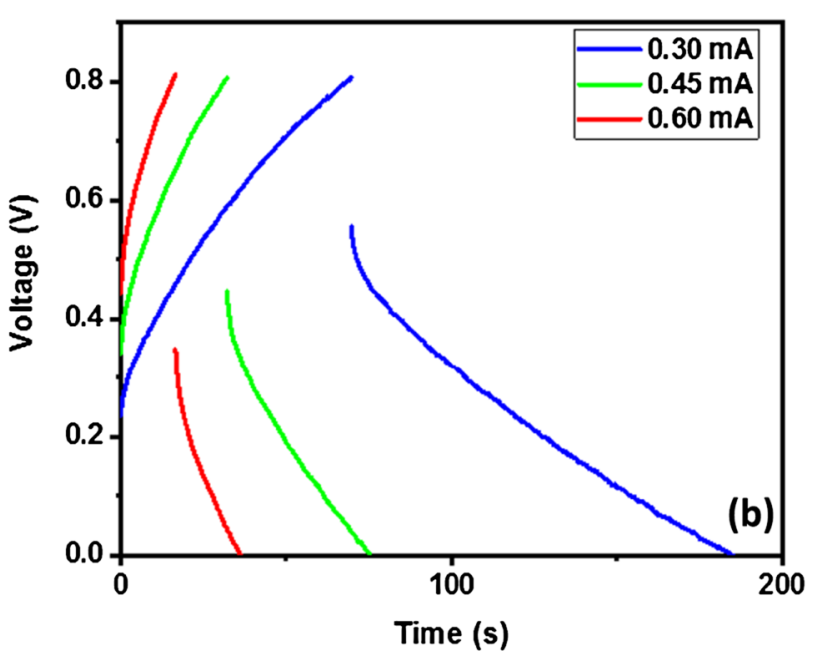

Fig. 5 a CV curves (at different scan rates) and b galvanostatic charge-discharge curves (at different charge density) for sample 75\% of GNP in $\mathrm{PPy} /(\mathrm{GNP} / \mathrm{CNT})$ 

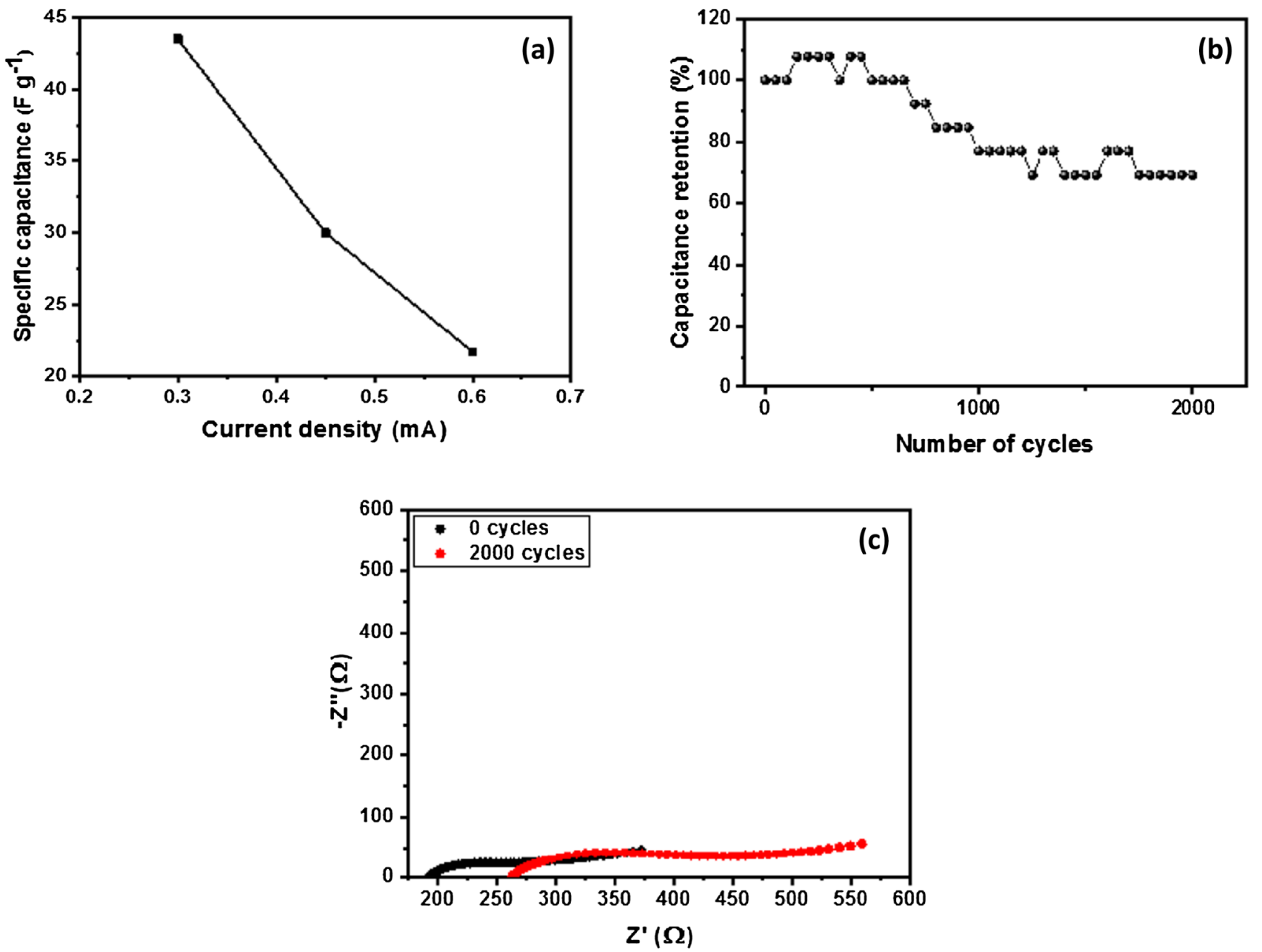

Fig. 6 a Specific capacitance of supercapacitor prepared with 75\% GNP, b capacitive retention of material after 2000 cycles and c impedance of samples performed before and after 2000 cycles of uses

capacitance after successive charge/discharge cycles. Result in Fig. $6 \mathrm{~b}$ shows a capacitive retention in order of $70 \%$ of initial value characterizing a moderate degradation of material after continuous reuse (2000 cycles). This process can be confirmed from impedance spectrum of samples. The Nyquist plot in Fig. $6 \mathrm{c}$ presents two specific regions: a depressed semicircle-characteristic of relaxation processes and a diffusive signature-at low frequency region, it is observed a linear branch with a typical slope in order of $45^{\circ}$. The progressive degradation of material (after 2000 uses) provokes a shift in the overall response in direction to higher impedance-it characterizes the increase in internal resistance of supercapacitor that is associated with variation in capacitive retention. The flexibility tests were performed according Refs. [17, 23]. The samples were successively bent from $0^{\circ}$ to $180^{\circ}$. As reported in the literature, these successive mechanical efforts result in the deterioration in the electrical/electrochemical properties of electrodes/supercapacitor in response of drop-off in the polymeric covering layer of electrodes [17, 23]. However, our results demonstrate that minimal changes are observed in the electrical resistance of electrodes (see Fig. 7a) in which is possible to verify variation in order of $10 \%$ after 1000 cycles of bending. Negligible variation in resulting capacitance (see Fig. 7b) is observed for corresponding mechanical process in supercapacitor prototypes, characterizing an important advantage for resulting prototypes as truly flexible and wearable devices.

\section{Conclusion}

The incorporation of GNP/CNT composites as covering layers of cotton yarn fibers and supporting layers for chemical polymerization produces flexible electrodes for use as wearable supercapacitors with improved electrochemical properties and prolonged action, as verified from good cyclability - high capacitive retention. The electrical 

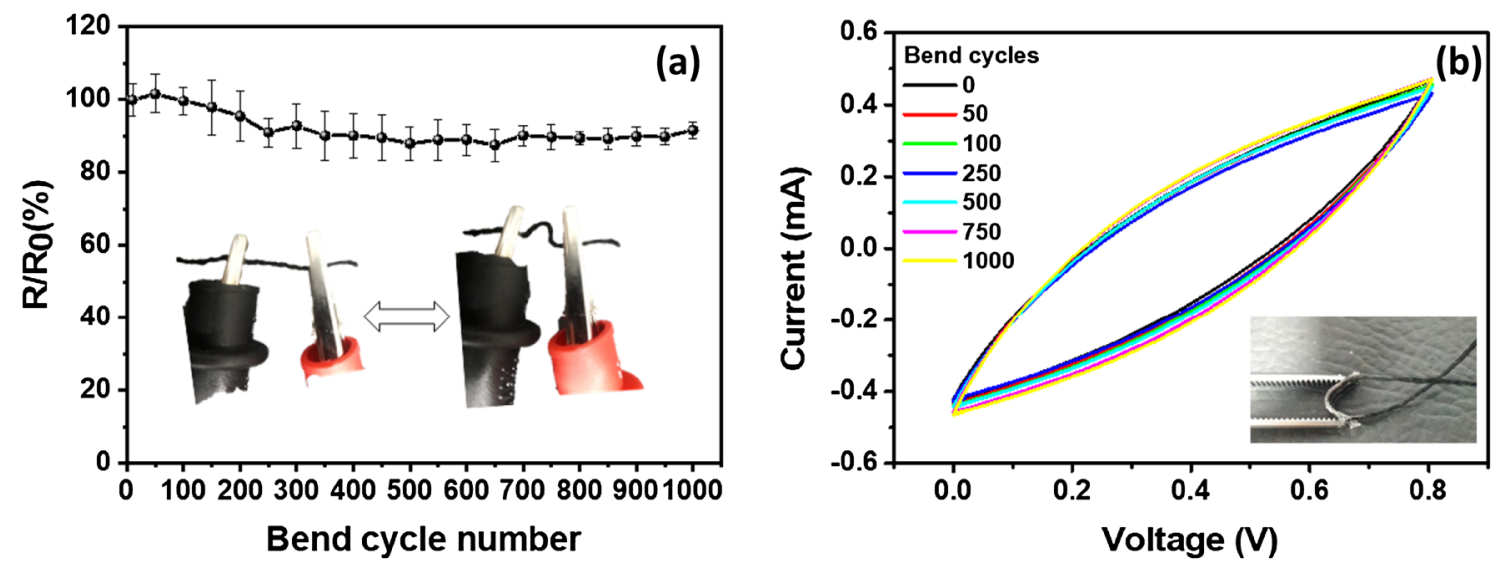

Fig. 7 a Relative variation of electrodes resistance under successive bend efforts and $\mathbf{b}$ corresponding variation in resulting capacitance for equivalent efforts on resulting supercapacitor (CV curves performed at scan rate of $20 \mathrm{mV} \mathrm{s}^{-1}$ )

conductivity and order/disorder ratio varied with relative composition of GNP/CNT and were explored in the determination of most adequate relative concentration of carbon derivatives for production of optimized supercapacitors. The comparison with corresponding systems produced with CNT confirmed superior results not only for specific capacitance but also for energy and power density for resulting devices.

Acknowledgements This work was partially supported by the Brazilian agencies FINEP, CAPES, FAPESB, FACEPE and CNPq.

\section{Compliance with ethical standards}

Conflict of interest On behalf of all authors, the corresponding author states that there is no conflict of interest.

\section{References}

1. Zhao Y, He X, Chen R, Liu Q, Liu J, Song D, Zhang H, Dong H, Li $\mathrm{R}$, Zhang M, Wang J (2018) Hierarchical $\mathrm{NiCO}_{2} \mathrm{~S}_{4} @ \mathrm{CoMoO}_{4}$ coreshell heterostructures nanowire arrays as advanced electrodes for flexible all-solid-state asymmetric supercapacitors. Appl Surf Sci 453:73-82. https://doi.org/10.1016/j.apsusc.2018.04.159

2. Garcia-Torres J, Crean C (2018) Ternary composite solid-state flexible supercapacitor based on nanocarbons/manganese dioxide/PEDOT:PSS fibres. Mater Des 155:194-202. https://doi. org/10.1016/J.MATDES.2018.05.070

3. Zhang Z, Zhang $P, Z$ hang $D$, Lin $H, C$ Chen $Y$ (2018) A new strategy for the preparation of flexible macroscopic graphene fibers as supercapacitor electrodes. Mater Des 157:170-178. https://doi. org/10.1016/J.MATDES.2018.07.043

4. Song P, Yang G, Lang T, Yong K-T (2019) Nanogenerators for wearable bioelectronics and biodevices. J Phys D Appl Phys 52:023002. https://doi.org/10.1088/1361-6463/aae44d

5. Liu H, Zhang Z, Ge J, Lin X, Ni X, Yang H, Yang Z (2019) A flexible conductive hybrid elastomer for high-precision stress/ strain and humidity detection. J Mater Sci Technol 35:176180. https://doi.org/10.1016/J.JMST.2018.09.006

6. Shi R, Lou Z, Chen S, Shen G (2018) Flexible and transparent capacitive pressure sensor with patterned microstructured composite rubber dielectric for wearable touch keyboard application. Sci China Mater 61:1587-1595. https://doi. org/10.1007/s40843-018-9267-3

7. Shih J-F, Yang S-Y, Chang C-C, Yang C-R (2018) Wearable sensors developed using a novel plastic metal material. Appl Phys A 124:799. https://doi.org/10.1007/s00339-018-2217-x

8. Qu S, Chen Y, Shi W, Wang M, Yao Q, Chen L (2018) Cottonbased wearable poly(3-hexylthiophene) electronic device for thermoelectric application with cross-plane temperature gradient. Thin Solid Films 667:59-63. https://doi.org/10.1016/J. TSF.2018.09.046

9. Chu X, Zhang H, Su H, Liu F, Gu B, Huang H, Zhang H, Deng W, Zheng $X$, Yang W (2018) A novel stretchable supercapacitor electrode with high linear capacitance. Chem Eng J 349:168175. https://doi.org/10.1016/J.CEJ.2018.05.090

10. Wang H-T, Liu Y-, Kang X-H, Wang Y-F, Yang S-Y, Bian S-W, Zhu $Q$ (2018) Flexible hybrid yarn-shaped supercapacitors based on porous nickel cobalt sulfide nanosheet array layers on gold metalized cotton yarns. J Colloid Interface Sci 532:527-535. https://doi.org/10.1016/J.JCIS.2018.08.006

11. Ren J, Ren RP, Lv YK (2018) Stretchable all-solid-state supercapacitors based on highly conductive polypyrrole-coated graphene foam. Chem Eng J 349:111-118. https://doi. org/10.1016/j.cej.2018.05.075

12. Zhao Y, He X, Chen R, Liu Q, Liu J, Yu LJ, Zhang H, Dong H, Zhang $M$, Wang J (2018) A flexible all-solid-state asymmetric supercapacitors based on hierarchical carbon cloth@CoMoO ${ }_{4} @ \mathrm{NiCo}$ layered double hydroxide core-shell heterostructures. Chem Eng J 352:29-38. https://doi.org/10.1016/J.CEJ.2018.06.181

13. Lima RMAP, Alcaraz-Espinoza JJ, da Silva FAG, de Oliveira HP (2018) Multifunctional wearable electronic textiles using cotton fibers with polypyrrole and carbon nanotubes. ACS Appl Mater Interfaces 10:13783-13795. https://doi.org/10.1021/ acsami.8b04695

14. Liu W, Song MS, Kong B, Cui Y (2017) Flexible and stretchable energy storage: recent advances and future perspectives. Adv Mater 29:1603436. https://doi.org/10.1002/adma.201603436

15. Dong L, Xu C, Li Y, Huang Z-H, Kang F, Yang Q-H, Zhao X (2016) Flexible electrodes and supercapacitors for wearable energy 
storage: a review by category. J Mater Chem A 4:4659-4685. https://doi.org/10.1039/c5ta10582j

16. Zhang $Y-Z$, Wang $Y$, Cheng $T$, Lai W-Y, Pang $H$, Huang W (2015) Flexible supercapacitors base on paper substrates: a new paradigm for low-cost energy storage. Chem Soc Rev 44:5181-5199. https://doi.org/10.1039/c5cs00174a

17. Wang J, Dong L, Xu C, Ren D, Ma X, Kang F (2018) Polymorphous supercapacitors constructed from flexible three-dimensional carbon network/polyaniline/ $\mathrm{MnO}_{2}$ composite textiles. ACS Appl Mater Interface 10:10851-10859. https://doi.org/10.1021/acsam i.7b19195

18. Dong L, Xu C, Yang Q, Fang J, Li Y, Kang F (2015) High-performance compressible supercapacitors based on functionally synergic multiscale carbon composite textiles. J Mater Chem A 3:4729-4737. https://doi.org/10.1039/c4ta06494a

19. Dong L, Liang G, Xu C, Ren D, Wang J, Pan Z-Z, Li B, Kang F, Yang Q-H (2017) Stacking up layers of polyaniline/carbon nanotube networks inside papers as highly flexible electrodes with large areal capacitance and superior rate capability. J Mater Chem A 5:19934-19942. https://doi.org/10.1039/c7ta06135h

20. Ren D, Dong L, Wang J, Ma X, Xu C, Kang F (2018) Facile preparation of high-performance stretchable fiber-like electrodes and supercapacitors. ChemistrySelect 3:4179-4184. https://doi. org/10.1002/slct.201702725

21. Dong L, Xu C, Li Y, Wu C, Jiang B, Yang Q, Zhou E, Kang F, Yang $\mathrm{QH}$ (2016) Simultaneous production of high-performance flexible textile electrodes and fiber electrodes for wearable energy storage. Adv Mater 28:1675-1681. https://doi.org/10.1002/ adma.201504747

22. Dong L, Xu C, Li Y, Pan Z, Liang G, Zhou E, Kang F, Yang QH (2016) Breathable and wearable energy storage based on highly flexible paper electrodes. Adv Mater 28:9313-9319. https://doi. org/10.1002/adma.201602541

23. Dong L, Liang G, Xu C, Liu W, Pan Z-Z, Zhou E, Kang F, Yang Q-H (2017) Multihierarchicval construction-induced superior capacitive performances of flexible electrodes for wearable energy storage. NanoEnergy 34:242-248. https://doi.org/10.1016/j. nanoen.2017.02.031

24. Ma Y, Wang Q, Liang X, Zhang D, Miao M (2018) Wearable supercapacitors based on conductive cotton yarns. J Mater Sci 53:14586-14597. https://doi.org/10.1007/s10853-018-2655-z

25. Kim JH, Choi C, Lee JM, de Andrade MJ, Baughman RH, Kim SJ (2018) Ag/ $\mathrm{MnO}_{2}$ composite sheath-core structured yarn supercapacitors. Sci Rep 8:13309. https://doi.org/10.1038/s4159 8-018-31611-2

26. Alcaraz-Espinoza JJ, de Oliveira HP (2018) Flexible supercapacitors based on a ternary composite of polyaniline/polypyrrole/ graphite on gold coated sandpaper. Electrochim Acta 274:200207. https://doi.org/10.1016/J.ELECTACTA.2018.04.063

27. Sadak O, Sundramoorthy AK, Gunasekaran S (2018) Facile and green synthesis of highly conducting graphene paper. Carbon 138:108-117. https://doi.org/10.1016/J.CARBON.2018.05.076

28. de Oliveira AHP, Nascimento MLF, de Oliveira HP (2016) Carbon nanotube@MnO @ @polypyrrole composites: chemical synthesis, characterization and application in supercapacitors. Mater Res 19:1080-1087. https://doi. org/10.1590/1980-5373-MR-2016-0347

29. de Oliveira AHP, de Oliveira HP (2014) Carbon nanotube/ polypyrrole nanofibers core-shell composites decorated with titanium dioxide nanoparticles for supercapacitor electrodes. J Power Sour 268:45-49. https://doi.org/10.1016/J.JPOWS OUR.2014.06.027

30. de Oliveira HP, Sydlik SA, Swager TM (2013) Supercapacitors from free-standing polypyrrole/graphene nanocomposites. J Phys Chem C 117:10270-10276. https://doi.org/10.1021/jp400 $344 \mathrm{u}$
31. Hao T, Sun J, Wang W, Yu D (2018) MWCNTs-COOH/cotton flexible supercapacitor electrode prepared by improvement onetime dipping and carbonization method. Cellulose 25:40314041. https://doi.org/10.1007/s10570-018-1829-9

32. Bo Y, Zhao Y, Cai Z, Bahi A, Liu C, Ko F (2018) Facile synthesis of flexible electrode based on cotton/polypyrrole/multi-walled carbon nanotube composite for supercapacitors. Cellulose 25:4079-4091. https://doi.org/10.1007/s10570-018-1845-9

33. Wei C, Xu Q, Chen Z, Rao W, Fan L, Yuan Y, Bai Z, Xu J (2017) An all-solid-state yarn supercapacitor using cotton yarn electrodes coated with polypyrrole nanotubes. Carbohydr Polym 169:50-57. https://doi.org/10.1016/J.CARBPOL.2017.04.002

34. Liu C, Cai Z, Zhao Y, Zhao H, Ge F (2016) Potentiostatically synthesized flexible polypyrrole/multi-wall carbon nanotube/cotton fabric electrodes for supercapacitors. Cellulose 23:637-648. https://doi.org/10.1007/s10570-015-0795-8

35. Shimpi NG, Hansora DP, Yadav R, Mishra S (2015) Performance of hybrid nanostructured conductive cotton threads as LPG sensor at ambient temperature: preparation and analysis. RSC Adv 5:99253-99269. https://doi.org/10.1039/C5RA16479F

36. Rosace G, Trovato V, Colleoni C, Caldara M, Re V, Brucale M, Piperopoulos E, Mastronardo E, Milone C, De Luca G, Plutino MR (2017) Structural and morphological characterizations of MWCNTs hybrid coating onto cotton fabric as potential humidity and temperature wearable sensor. Sens Actuators B Chem 252:428-439. https://doi.org/10.1016/J.SNB.2017.05.175

37. Bharath SP, Manjanna J, Javeed A, Yallappa S (2015) Multi-walled carbon nanotube-coated cotton fabric for possible energy storage devices. Bull Mater Sci 38:169-172. https://doi.org/10.1007/ s12034-014-0829-0

38. Gupta S (2006) Template-free synthesis of conducting-polymer polypyrrole micro/nanostructures using electrochemistry. Appl Phys Lett 88:063108. https://doi.org/10.1063/1.2168688

39. Xiao X, Li T, Yang P, Gao Y, Jin H, Ni W, Zhan W, Zhang X, Cao Y, Zhong J, Gong L, Yen W-C, Mai W, Chen J, Huo K, Chueh Y-L, Wang ZL, Zhou J (2012) Fiber-based all-solid-state flexible supercapacitors for self-powered systems. ACS Nano 6:9200-9206. https://doi.org/10.1021/nn303530k

40. Yuan L, Lu X-H, Xiao X, Zhai T, Dai J, Zhang F, Hu B, Wang X, Gong L, Chen J, Hu C, Tong Y, Zhou J, Wang ZL (2012) Flexible solidstate supercapacitors based on carbon nanoparticles $/ \mathrm{MnO}_{2}$ nanorods hybrid structure. ACS Nano 6:656-661. https://doi. org/10.1021/nn2041279

41. Zhao C, Wang C, Yue Z, Shu K, Wallace GG (2013) Intrinsically stretchable supercapacitors composed of polypyrrole electrodes and highly stretchable gel electrolyte. ACS Appl Mater Interfaces 5:9008-9014. https://doi.org/10.1021/am402130j

42. Duch J, Mazur M, Golda-Cepa M, Podobiński J, Piskorz W, Kotarba A (2018) Insight into the modification of electrodonor properties of multiwalled carbon nanotubes via oxygen plasma: surface functionalization versus amorphization. Carbon 137:425-432. https://doi.org/10.1016/J.CARBON.2018.05.059

43. Ahmed S, Rafat M, Singh MK, Hashmi AS (2018) A free-standing, flexible PEDOT:PSS film and its nanocomposites with graphene nanoplatelets as electrodes for quasi-solid-state supercapacitors. Nanotechnology 29:395401. https://doi.org/10.1088/13616528/aad0b8

44. da Silva FAG, de Araújo CMS, Alcaraz-Espinoza JJ, de Oliveira HP (2018) Toward flexible and antibacterial piezoresistive porous devices for wound dressing and motion detectors. J Polym Sci Part B Polym Phys 56:1063-1072. https://doi.org/10.1002/ polb.24626

45. Alcaraz-Espinoza JJ, de Melo CP, de Oliveira HP (2017) Fabrication of highly flexible hierarchical polypyrrole/carbon nanotube on eggshell membranes for supercapacitors. ACS Omega 2:2866-2877. https://doi.org/10.1021/acsomega.7b00329 
46. Zhou Y, Xie Y (2018) Enhanced electrochemical stability of carbon quantum dots-incorporated and ferrous-coordinated polypyrrole for supercapacitor. J Solid State Electrochem 22:2515-2529. https://doi.org/10.1007/s10008-018-3964-5

47. Lim SP, Pandikumar A, Lim YS, Huang NM, Lim HN (2015) In situ electrochemically deposited polypyrrole nanoparticles incorporated reduced graphene oxide as an efficient counter electrode for platinum-free dye-sensitized solar cells. Sci Rep 4:5305. https ://doi.org/10.1038/srep05305
Publisher's Note Springer Nature remains neutral with regard to jurisdictional claims in published maps and institutional affiliations. 